\title{
Particle Acceleration in three dimensional Reconnection Regions: A New Test Particle Approach
}

\author{
Rüdiger Schopper \\ Geophysical Institute, University of Alaska Fairbanks, Fairbanks AK 99775, USA \\ Guido T. Birk \\ Department of Astronomy, University of Toronto, Toronto, Ontario M5S 1A\%, Canada \\ Harald Lesch \\ Institut für Astronomie und Astrophysik, LMU-München, D-81679 München, Germany
}

\begin{abstract}
Magnetic Reconnection is an efficient and fast acceleration mechanism by means of direct electric field acceleration parallel to the magnetic field. Thus, acceleration of particles in reconnection regions is a very important topic in plasma astrophysics. This paper shows that the conventional analytical models and numerical test particle investigations can be misleading concerning the energy distribution of the accelerated particles, since they oversimplify the electric field structure by the assumption that the field is homogeneous. These investigations of the acceleration of charged test particles are extended by considering three-dimensional field configurations characterized by localized field-aligned electric fields. Moreover, effects of radiative losses are discussed. The comparison between homogeneous and inhomogeneous electric field acceleration in reconnection regions shows dramatic differences concerning both, the maximum particle energy and the form of the energy distribution.
\end{abstract}

PACS numbers: 52.20 ; 52.65.C ; D95.30 ; 95.30Q

\section{Introduction}

The acceleration of charged particles in space and astrophysical plasmas is a matter of debate in contemporary plasma astrophysics (cf. reviews and 2 ). Beside the "standard" acceleration mechanisms as shock waves

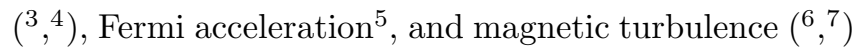
acceleration by magnetic field-aligned electric fields receives more and more attention as it offers the capability for efficient and fast energization of relativistic particles (3,8). The formation and amplification of such electric fields in the context of magnetic reconnection processes has been discussed for different cosmical plasmas and parameter regimes $(10,0,11,12,13)$. Since magnetic reconnection can be regarded as an unavoidable dissipation and relaxation process in magnetized plasmas under the influence of externally driven shear motions, the principal importance of magnetic reconnection electric fields is now widely accepted.

Analytical and numerical studies have significantly improved our understanding of energetic particle trajectories in reconnection cenfigurations without (14, 15, $16,27,18,19)$ and with $(20,21,22)$ a magnetic field component parallel to the reconnection current sheet. Nevertheless, the particle trajectories and, in particular, the actual energy gains of accelerated charged particles in the reconnection current sheets prove to be still a crucial matter of debate, since the studies mentioned above have been performed for idealized analytical electric and magnetic field configurations which differ significantly from the three-dimensional configurations characterized by localized field-aligned electric fields as observed, e.g. in the solar corona (cf.23) and the Earth's magnetosphere (cf.24). Test particle simulations of ion (25) and electron (26) injection in a high-beta magnetospheric substorm configurations have shown that the consideration of more complicated $3 \mathrm{~d}$ field configurations rather than idealized $2 \mathrm{D}$ ones is crucial for the understanding of particle acceleration in reconnection regions. In this contribution we study the acceleration of charged particles up to relativistic energies in reconnection regions. The electromagnetic fields of the reconnection region are modeled by 3D-magnetohydrodynamic (MHD) simulations of a reconnection zone in a current carrying magnetic flux tube. Since the astrophysical evidence for the existence of relativistic particles relies only on the electromagnetic radiation emitted by of these particles, we calculate the motion of high-energy particles by means of relativistic test particle simulations including radiative forces due to inverse Compton scattering and synchrotron radiation. We consider our study as a step forward to a self-consistent description of particle acceleration in quite realistic reconnection configurations.

The manuscript is organized as follows: The next section presents the motivation for our new approach. Sec. III describes the electromagnetic field configuration 
we used and how they are obtained from MHD simulations. These fields represent the external force field, to be supplemented by radiative forces, for the relativistic test particle calculations, described in Sec. IV. The results of these test particle simulations are compared with the analytical and numerical X-type configuration of the reconnection magnetic field in Sec. V. Sec. VI is devoted to a discussion of our results.

\section{Motivation for our new Approach}

Particle acceleration in magnetic reconnection zones has been extensively discussed and studied analytically and numerically (we refer to the analytical papers 14,27,2,22, and references therein); numerical treatments can be found in 16.29, and references therein). These investigations rely on one common assumption The homogeneity of the electric field. The authors have used an analytical formulation of the electric field configuration and therefore the calculations are highly idealized. The field configurations found in the papers mentioned above are nearly identical and can be written as

$$
\boldsymbol{B}(\boldsymbol{r})=B_{0}\left(\begin{array}{c}
\frac{y}{L_{x}} \\
\frac{x}{L_{y}}+\xi \\
1
\end{array}\right) \quad \boldsymbol{E}(\boldsymbol{r})=E_{0}\left(\begin{array}{l}
0 \\
0 \\
1
\end{array}\right) .
$$

The process of reconnection in two dimensions is still subject of intense scientific investigation up to the present day. Besides first analytical approaches (30;31) investigations of the much more complex $3 \mathrm{D}$ reconnection process are restricted to numerical modeling. In a $2 \mathrm{D}$ description the $\mathrm{X}$-point configuration

$$
\boldsymbol{B}(\boldsymbol{r})=B_{0}\left(\begin{array}{c}
\frac{y}{L_{x}} \\
\frac{x}{L_{y}} \\
0
\end{array}\right)
$$

that describes a hyperbolic magnetic field with a neutral line in the center $(\boldsymbol{B}(0,0, z)=0)$, has become the standard model of reconnection. With Ampre's Law

$$
\frac{4 \pi}{c} \boldsymbol{j}=\boldsymbol{\nabla} \times \boldsymbol{B}
$$

Ohm's Law

$$
\eta \boldsymbol{j}=\boldsymbol{E}+\boldsymbol{v} / c \times \boldsymbol{B}
$$

and invariance in one dimension, say $\partial_{z} \cdot=0$, one can easily derive the electric field

$$
\boldsymbol{E}=\eta \frac{B_{0} c}{4 \pi}\left(\frac{1}{L_{x}}-\frac{1}{L_{y}}\right)\left(\begin{array}{l}
0 \\
0 \\
1
\end{array}\right)-\boldsymbol{v} / c \times \boldsymbol{B} .
$$

If one assumes a constant resistivity and that the convective term $\boldsymbol{v} / c \times \boldsymbol{B}$ is small as compared to the first term in (Equ. 5) in the localized reconnection zone the constant, homogeneous, infinitely extended electric field is given by

$$
\boldsymbol{E}=\eta \frac{B_{0} c}{4 \pi}\left(\frac{1}{L_{x}}-\frac{1}{L_{y}}\right)\left(\begin{array}{l}
0 \\
0 \\
1
\end{array}\right) .
$$

Such an electric field is, without question, unphysical. In case of a $2 \mathrm{D}$ reconnection calculation this is not a severe problem, because the electric field is never used as a real physical quantity. For this reason one can argue that it is merely a mathematical auxiliary variable and only the magnetic field is needed to describe the complete electromagnetic configuration.

However, this $\mathrm{X}$-type field is a valid approximation of the inner part of a reconnection region around the neutral line. Only the application of this idealized configuration in the context of particle acceleration gives rise to serious problems. The electric field is the source of energy for the accelerated particles and exactly this very reason for acceleration is, as stated above, unphysical. At this point, we should ask how sensible are the results concerning particle energy spectra, duration of acceleration etc., derived from an idealized, homogeneous electrical field? For example, there are particle trajectories

$$
\boldsymbol{r}=\left(\begin{array}{c}
0 \\
0 \\
z
\end{array}\right) \quad \boldsymbol{p}=\left(\begin{array}{c}
0 \\
0 \\
p
\end{array}\right) \quad \boldsymbol{B}=B_{0}\left(\begin{array}{c}
\frac{y}{L_{x}} \\
\frac{x}{L_{y}} \\
1
\end{array}\right)
$$

for which particles will gain infinite energy. This comes from the fact that the electric field lacks any dependence on the invariant direction.

In order to accelerate articles efficiently a strong guiding component $B_{z}(27)$ is needed. With such a strong $B_{z}$ component the magnetic field is almost parallel to the electric field in a region $B_{z}^{2} \gg B_{x}^{2}+B_{y}^{2}$ around the neutral line. In addition, this guiding component captures the particles in the zone, at least as long as their gyroradius $r_{\text {ce }}$ is smaller than the width of the reconnection zone. These two facts guarantee the efficient acceleration described in 27 . On the other hand this configuration in the center of a X field with strong $B_{z}$ resembles remarkably that of a simple capacitor. Any particle following the field lines is accelerated exactly linear, like in a capacitor. The energy gained by such a particle is directly given by

$$
\Delta \mathcal{E}=q E_{z} \Delta L
$$

$\Delta \mathcal{E}$ denotes the energy gain and $q$ is the charge of the particle that is accelerated along the magnetic field line over the distance $\Delta L$. 


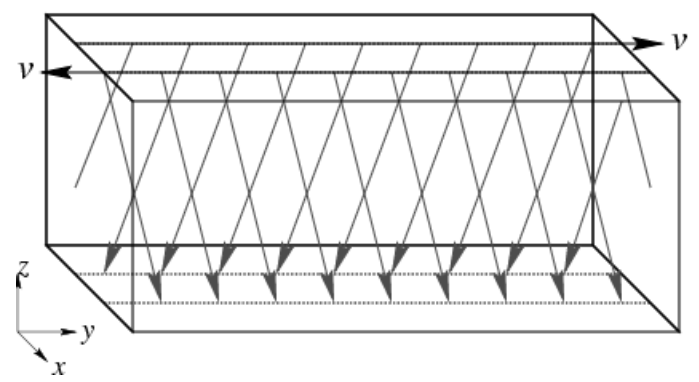

Fig. 1. Sketch of the generic initial configuration.

The energy gain does not depend on the detail of the reconnection process, but on the geometry of the acceleration region only.

Moreover, one has to take into account the convective electric field components $E_{x}$ and $E_{y}$, which are completely neglected by most authors, though they naturally arise in realistic descriptions of reconnection zones. Such fields are generated by external shear flows which are the macroscopic generator of electromagnetic energy converted partly into particle kinetic energy in the localized dissipative reconnection zones. Calculations with three-dimensional magnetohydrodynamic (3D-MHD) codes clearly show (e.g.32) that those components are often much stronger than the field line parallel electric field $E_{\|}$. The precise influence of those components is not known, nevertheless we should not neglect them strait from the beginning, without knowing anything about the strength of their influence. We speculate that such convective electric fields can filter out particles from an efficient acceleration due to their strength and structure and dependent on the initial positions of the particles. In other words, the form of the energy distribution may be closely connected to these electric fields.

The above questions convinced us to approach again this well known and well studied phenomenon of particle acceleration in magnetic reconnection zones. Contrary to other investigations, we use a field configuration, which is produced with the help of a full 3D-MHD code.

\section{The 3D-MHD Simulations of Magnetic Recon- nection}

The MHD framework for the particle simulations of electron acceleration in reconnection regions is -built up by means of a three-dimensional MHD code 33 . It is motivated by previous works on reconnection in the coptextofplanetary magnetosphere-ionosphere coupling $(11,13,22,32,44)$ and of active galactic nucleit22. However, the situation we have in mind (cf. Fig. 1) is quite general and may be regarded as a rather generic recon- nection configuration that arises when MHD shear flows working as electromagnetic generators sheared magnetic fields. For convenience, we start from a two-dimensional force-free magnetic field

$$
\boldsymbol{B}=B_{y 0} \tanh (x) \mathbf{e}_{y}-\sqrt{B_{z 0}^{2}+\frac{B_{y 0}^{2}}{\cosh ^{2}(x)}} \mathbf{e}_{z} .
$$

We choose for the constant main component and the shear component of the magnetic field $B_{z 0}=5$ and $B_{y 0}=1$ in normalized units. The plasma is chosen to be isothermal as well as homogeneous, initially. We note that, alternatively, an appropriate equilibrium that includes thermal pressure forces could be realized. This ideal equilibrium is perturbed by

$$
v_{y}(x, z)=v_{y 0} \frac{\tanh (2 x)}{\cosh ^{2}\left(\frac{x}{3}\right)} \exp \left(-\frac{z}{6}\right) .
$$

As a boundary condition for $t>0$ we use

$$
v_{y}\left(x, z=z_{\min }\right)=v_{y 0} \frac{\tanh (2 x)}{\cosh ^{2}\left(\frac{x}{3}\right)} .
$$

These velocity perturbation with an amplitude chosen as $v_{y 0}=0.5 \%$ of the Alfvén velocity mimics some external convective plasma motion which leads to a further shear of the magnetic field and an increase of the field-aligned current density. The associated magnetic perturbation is transported along the $z$-component of magnetic field via shear Alfvén waves. Here, we follow the general concept that plasma systems that are compelled by such external forces when some critical parameter(s) is (are) exceeded non-linearly develop into a state of lower energy thereby releasing stored free energy in form of dissipative plasma heating and particle acceleration.

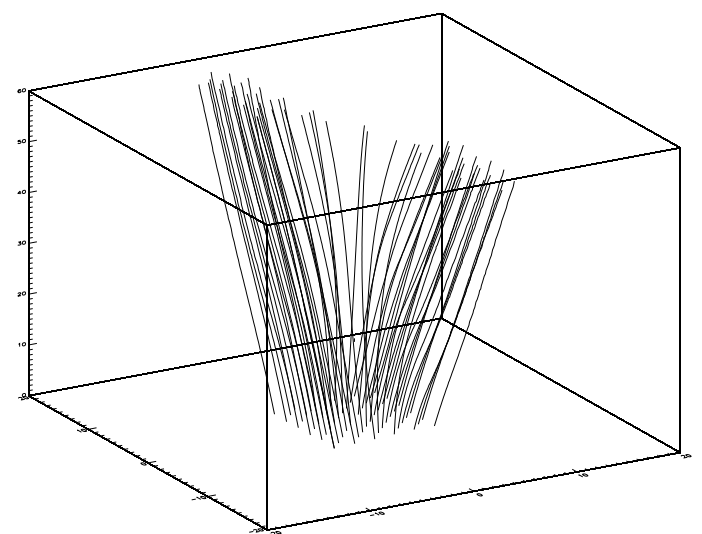

Fig. 2. Some characteristic magnetic fieldlines after $t=120 \tau_{\mathrm{A}}$ 

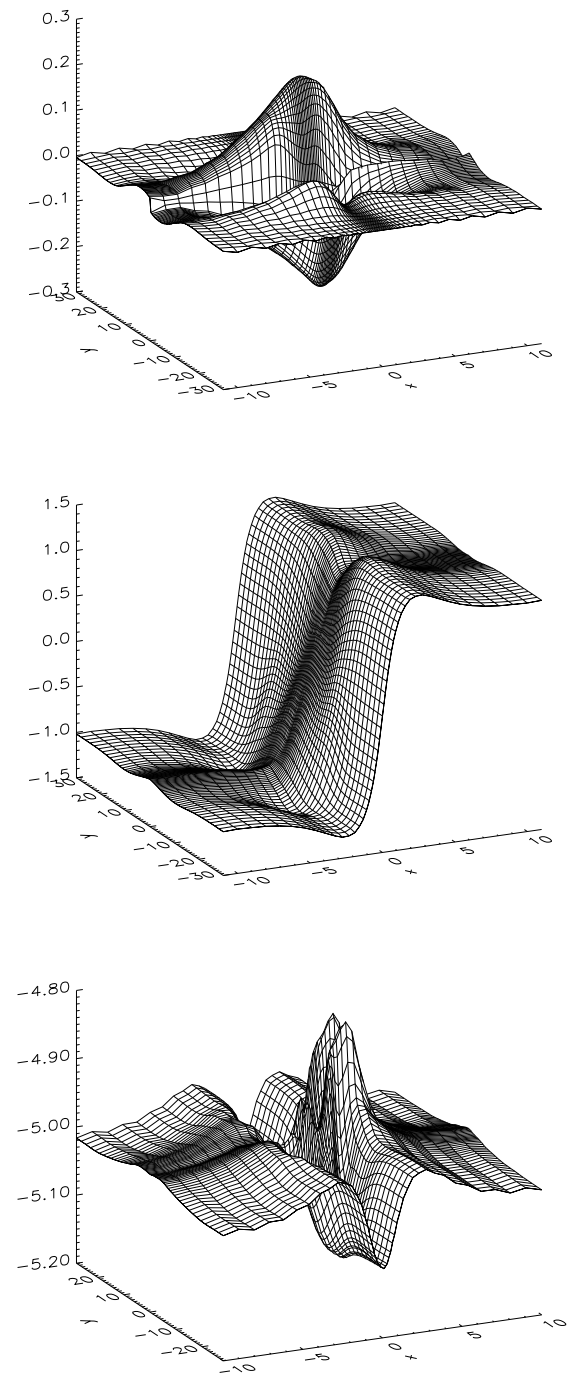

Fig. 3. Snapshot of the $x-, y$ - and $z$-component (from top to bottom) of the reconnection magnetic field at the height of the central reconnection region after $t=120 \tau_{\mathrm{A}}$.

In the considered configuration the magnetic fieldaligned current density plays the role of the critical parameter. When the current density exceeds a critical value (we choose a marginal current density at the beginning) a current dependent resistivity, which is localized in the $y$ - and $z$-direction is switched on. This violation of ideal $\mathrm{Ohm}$ 's law starts the magnetic reconnection process (cf 9 ). Fortunately, for our purpose we have not to dwell on the specific mature of the finite resistivity, may it be microturbulent 35 dissipation or particle inertia 36 or whatever. For the MHD simulations we choose the following technical details:
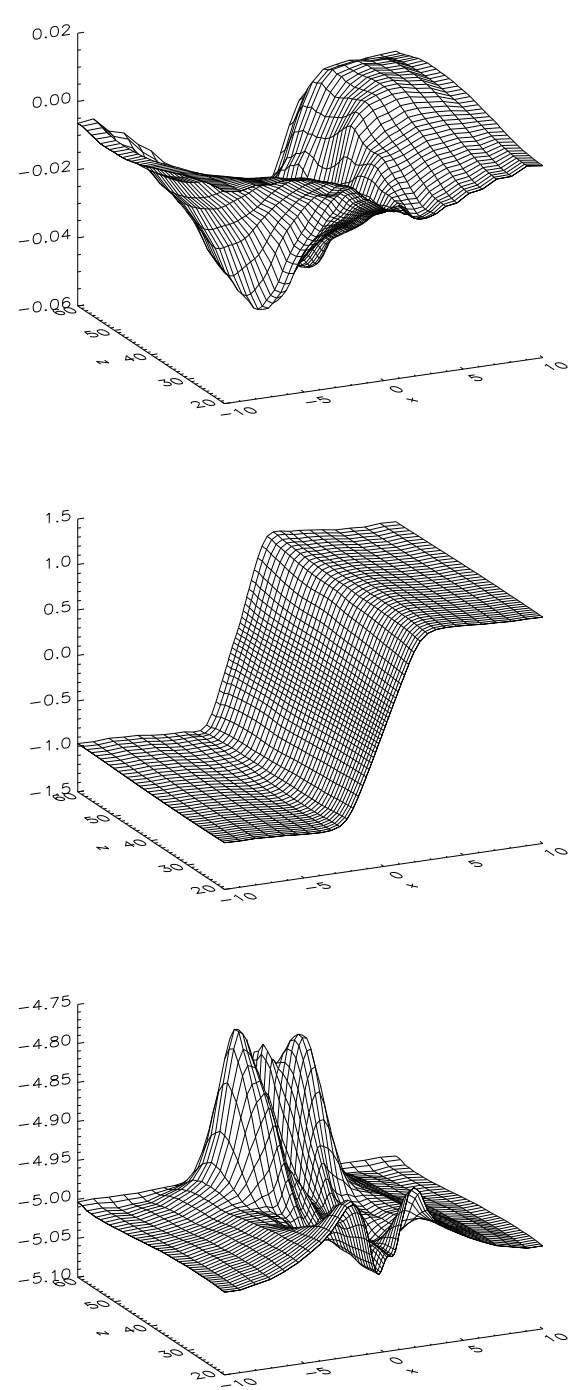

Fig. 4. Snapshot of the $x$-, $y$ - and $z$-component (from top to bottom) of the reconnection magnetic field at the $y=0$-half-plane after $t=120 \tau_{\mathrm{A}}$.

The dimensions of the numerical box are given by $x \in[-10,10], y \in[0,30]$, and $z \in[0,60]$ in normalized units. We make use of line symmetry as a boundary conditions at $y=0$, i.e. the $x$ - and $y$-components of the magnetic field and the velocities are chosen antisymmetric whereas the $z$-components of these quantities are chosen symmetric about $y=0$. Boundary conditions in $x$ are chosen as symmetric and in $z$ as well as at $y=y_{\max }$ we use extrapolation. The simulations are carried out with 49 grid points in the $x$ - direction, 39 grid points in the $y$-direction and 105 grid point in the $z$-direction. 

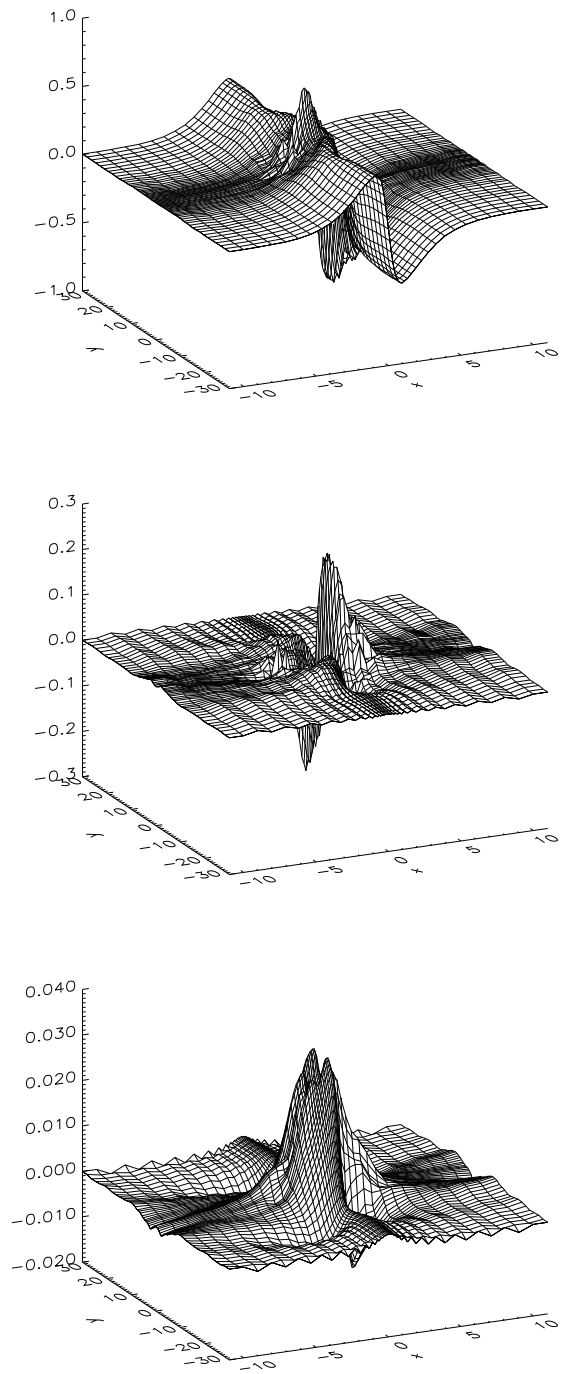

Fig. 5. Snapshot of the $x$-, $y$ - and $z$-component (from top to bottom) of the reconnection electric field at the height of the central reconnection region after $t=120 \tau_{\mathrm{A}}$.

The numerical grid is a non-uniform one with a maximum resolution of 0.05 in the $x$-direction, 0.4 in the $y$-direction, and 0.2 in the $z$-direction.

Some characteristic fieldlines are shown in Fig. 2 to give an idea of the overall structure of the magnetic field. In Figs. 3 to 6 we show the Cartesian components of the electric and magnetic fields which are chosen as an input for the particle simulation studies. The figures show the fields after $t=120 \tau_{\mathrm{A}}$ where $\tau_{\mathrm{A}}$ is the characteristic Alfvén velocity of the system. At this state of the temporal evolution the reconnection process can be considered as quasi-stationary, i.e. the magnetic flux is as rapidly piled up by the shear flow as it is annihilated by
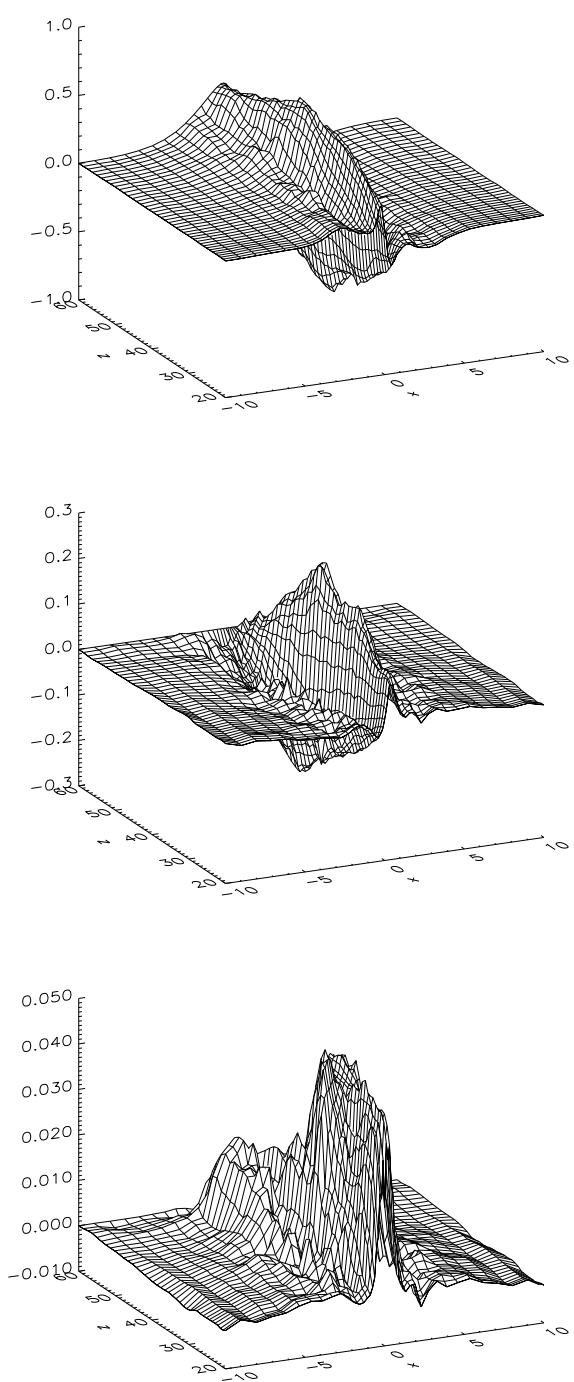

Fig. 6. Snapshot of the $x-, y$ - and $z$-component (from top to bottom) of the reconnection electric field at the $y=0$-half-plane after $t=120 \tau_{\mathrm{A}}$.

magnetic reconnection.

The $x$-component of the magnetic field results from the shear flow and from the divergent reconnection flow (Fig. 3, top panel) and (Fig. 1, top panel). The $y$ component of the magnetic field (middle panels of Fig. 3 and Fig. 3) is associated with the current sheet and modified by reconnection convergent flows $\left(v_{x}(x)\right)$. The $z$ component (lower panels of Fig. 3 and Fig. 3) shows a rather moderate variation due to the reconnection process. The three-dimensional electric vector field at the height of the central reconnection region is illustrated in Fig. 5. The convective $x$-component (top panels of Fig. 5 and Fig. (6) is the result of both the applied sheared flow 
and the divergent reconnection flow whereas the convective $y$-component (middle panels of Fig. 5 and Fig. 6) is due to the convergent reconnection flow. The convective electric fields are about one order of magnitude higher than the $z$-component of the electric field (lower panels of Fig. 5 and Fig. 6) which is caused by the finite anomalous electric resistivity $E_{z} \sim \eta j_{z} \approx E_{\|} \approx \eta j_{\|} \cdot E_{z}$ is localized in the $x$ - and $y$-direction (we have chosen for the resistivity profile $\eta \sim \cosh (y(i v) / 2$. $))$ and is most important for the acceleration of charged particles.

It is mainly responsible for the non-vanishing elpmgated generalized field-aligned electric potential $(9,37)$ $U=-\int E_{\|} d s$ (where the integration is extended over the arch length $s$ ). Such potentials are observed, e.g. as "auroral potential structures" in the Earth's magnetosphere 38 which are responsible for the acceleration of auroral electrons. The three-dimensional electric and magnetic vector field are used as an input for the test particle simulation studies of relativistic electrons as discussed in the next chapter. Note, that these rather complex fields, which are due to shear Alfvén waves, shear motion and reconnection dynamics, differ significantly from idealized configurations usually discussed in the literature. We consider the electromagnetic field shown as a quite typical reconnection configuration in systems that are characterized by shear flows. Since it represents the self-consistent reconnection dynamics it can be qualified as a realistic configuration in comparison to idealized two-dimensional ones.

\section{Particle Acceleration}

We use the fields described in Sec. III for electron acceleration. By means of a fully relativistic test particle code, including energy losses due to synchrotron emission (SY) and inverse Compton scattering (IC) we analyze the fate of the relativistic particles. The term "test particle" in this context means that any back-reaction of particles on the electromagnetic field configuration is neglected. As long as only a fraction of particles is actually accelerated, i.e. as long as the particles leave the acceleration zone on time scales much shorter than the Alfvénic time this is a very good approximation, which is always fulfilled during our simulations, since the particles are efficiently accelerated along the magnetic field line and move with almost the velocity of light. Moreover, no interaction between the particles is included.

For the integration of the relativistic equations of motion

$$
\frac{\mathrm{d} \boldsymbol{r}}{\mathrm{dt}}=\boldsymbol{v} \quad \frac{\mathrm{d} \boldsymbol{p}}{\mathrm{dt}}=\boldsymbol{F} \quad \boldsymbol{p}=\frac{m_{0} \boldsymbol{v}}{\sqrt{1-\left(\frac{v}{c}\right)^{2}}}
$$

we use the classical fourth order Runge-Kutta algorithm together with an adaptive stepsize control 39 . Our 3DMHD fields are only known on a discrete 3D grid. For this reason, we have to interpolate these fields for positions in between by means of a simple linear interpolation algorithm.

As described in Sec. III the electromagnetic fields can be regarded as quasi-stationary after some hundred Alfvnic times $t \simeq 120 \tau_{\mathrm{A}}$. Moreover, the test electrons are accelerated on a very short time scale $t_{\mathrm{acc}} \simeq 10^{-2} \tau_{\mathrm{A}}$ (cf.40). For this reason there is no need to consider the precise dynamic evolution of the MHD fields. We can use them as static background fields without any temporal changes and this is also a very good approximation. In addition to the Lorentz force $\boldsymbol{F}_{\mathrm{L}}=q(\boldsymbol{E}+\boldsymbol{v} / c \times \boldsymbol{B})$ our code considers the complete loss force 11

$$
\begin{aligned}
\boldsymbol{F}_{\mathrm{Rad}} & =\frac{2 q^{3} \gamma}{3 m c^{3}}\left\{\frac{\mathrm{d} \boldsymbol{E}}{\mathrm{dt}}+\frac{1}{c} \boldsymbol{v} \times \frac{\mathrm{d} \boldsymbol{B}}{\mathrm{dt}}\right\} \\
& +\frac{2 q^{4}}{3 m^{2} c^{4}}\left\{\frac{1}{q} \boldsymbol{F}_{\mathrm{L}} \times \boldsymbol{B}+\frac{1}{c} \boldsymbol{E}(\boldsymbol{v} \cdot \boldsymbol{E})\right\} \\
& -\frac{2 q^{4} \gamma^{2}}{3 m^{2} c^{5}} \boldsymbol{v}\left\{\frac{1}{q^{2}} F_{\mathrm{L}}^{2}-\frac{1}{c^{2}}(\boldsymbol{E} \cdot \boldsymbol{v})^{2}\right\},
\end{aligned}
$$

where $q, m, \boldsymbol{v}$ and $\gamma$ denote the charge, the mass, the velocity and the Lorentz factor of a particle, respectively. This loss force describes all particle-field interactions, inclusive synchrotron radiation (SY) and inverse Compton scattering (IC). SY presents the radiation of relativistic electrons, gyrating around the magnetic field, i.e. it influences the particle momentum perpendicular to the magnetic field. If an acceleration process injects particles merely along the field lines these particles undergo no synchrotron losses. However, since we inject particles isotropically from all directions, the particles will have finite pitch angles at the beginning. Even for very anisotropic energy distributions in the sense that the particles move only parallel to the magnetic field lines particles may suffer radiative losses caused by inverse Compton scattering, if an intense photon bath is present in the environment of the acceleration zone. The radiation mechanisms depend on the energy of the particles and the energy density of the magnetic field $U_{\mathrm{Mag}} \propto B^{2}$ (SY) or the photons $U_{\text {Rad }}(\mathrm{IC})$, respectively,

Qualitatively, it is clear that relatively strong (in terms of efficiency and short time scales) particle acceleration via magnetic reconnection requires significant magnetic field strengths. Since astrophysical plasmas are highly conducting media, strong magnetic fields are related with either fast plasma motions or high plasma pressure and temperature, i.e intense radiation fields. Such conditions are typical, e.g., for compact astrophysical objects, as neutron stars or black holes and magnetospheres of 
young stellar objects. Often the magnetic field energy density and the radiation energy density are of comparable order, or at least proportional to each other. This quality is again connected with the high electric conductivities of astrophysical plasmas on macroscopic spatial scales. If plasma is accelerated due to some radiation pressure, the magnetic field is strongly coupled to the plasma motion and is amplified by field line compression and twisting. The plasma temperature is directly related to the radiation temperature. Thus, a significant variation of the emitted radiation will consequently produce varying plasma temperatures and pressures, which finally leads to magnetic field amplification (e.g.42). Thus, the energy losses of the accelerated particles can be handled by a simple scaling $U_{\mathrm{Mag}} \propto U_{\mathrm{rad}}$.

\section{Comparison}

We use our code for the calculation of electron energy spectra which one would expect to be the final energy distributions in some selected configuration. In order to perform this we calculate the trajectories of more than 8000 electrons, which start at random places with a random momentum and determine their final energies, when they leave the numerical bounding box of the MHD fields. The initial positions are equally distributed in the whole reconnection region. The initial momentum of the electrons is chosen isotropic with absolute values that correspond to a Maxwellian distribution of the initial energy.

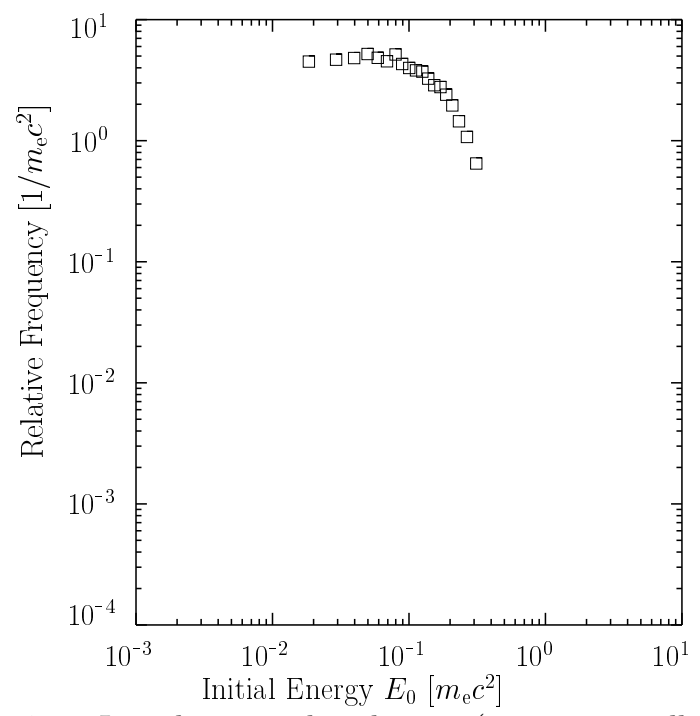

Fig. 7. Initial energy distribution (common to all simulations).

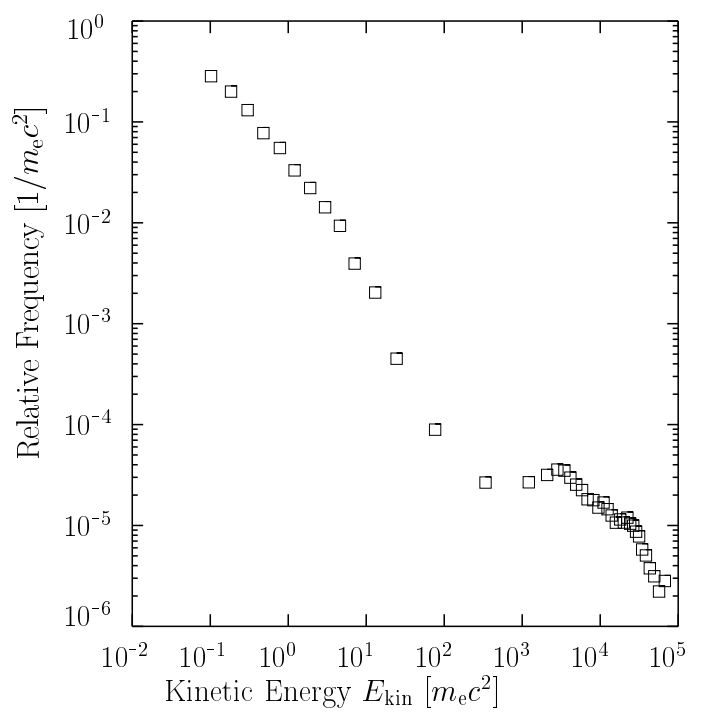

Fig. 8. Final energy distribution, calculated with realistic 3-D-MHD fields.

In Fig. 7 the initial energy distribution of the electrons is shown. Here and in all following plots "Relative Frequency" is the normed $\left(\int d E=1\right)$ percentage of particles per energy interval. All runs start from this distribution. Fig. 8 shows the final energy distribution of electrons accelerated in our quite realistic 3D-MHD fields (see Sec. III). Most electrons are accelerated due to the strong guiding component $B_{z}$. Energies in the range from $10^{-1} m_{\mathrm{e}} c^{2}$ to nearly $10^{5} \mathrm{~m}_{\mathrm{e}} c^{2}$ are seen. One first remarkable fact is the existence of electrons nearly without any energy gain. A second interesting feature is the obvious depression in the middle of this spectrum.

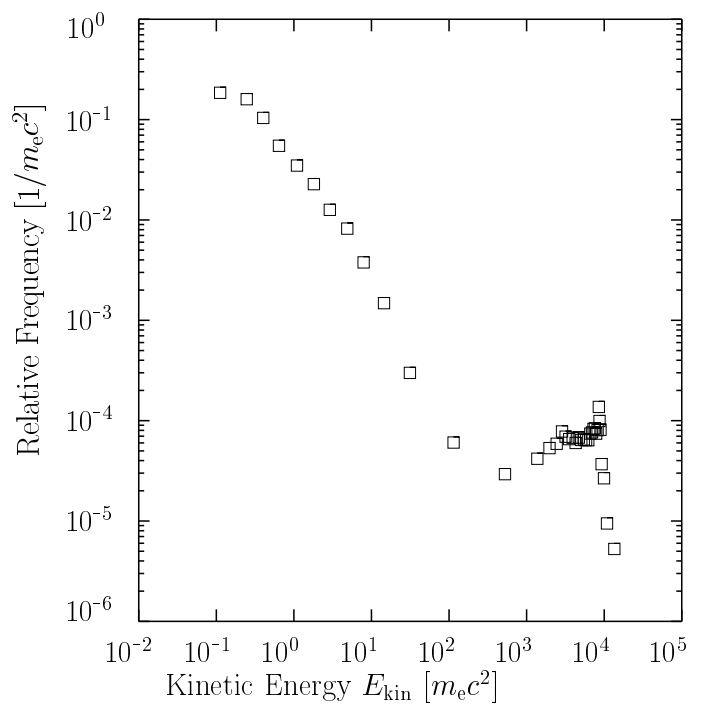

Fig. 9. Final energy distribution, calculated with realistic 3-D-MHD fields and consideration of loss processes. $U_{\text {Rad }} \sim 10 \cdot U_{\text {Mag. }}$. 


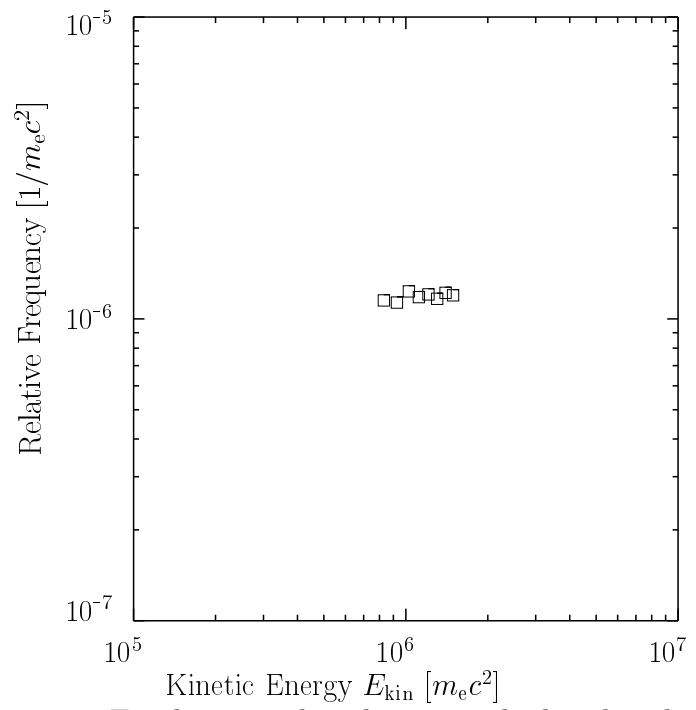

Fig. 10. Final energy distribution, calculated with a simplified, homogeneous electric field and the realistic magnetic field.

It seems as if there exists some sort of selection process, which does not allow a particle to achieve an energy between $10^{2} m_{\mathrm{e}} c^{2}$ and $10^{3} m_{\mathrm{e}} c^{2}$. This might indicate that there are unstable trajectories, which lead exactly to this energy range. The high energetic end of the particle distribution is determined by the thickness of the resistive region. Only in the region of finite resistivity a parallel electric field is present, which can accelerate the particles efficiently.

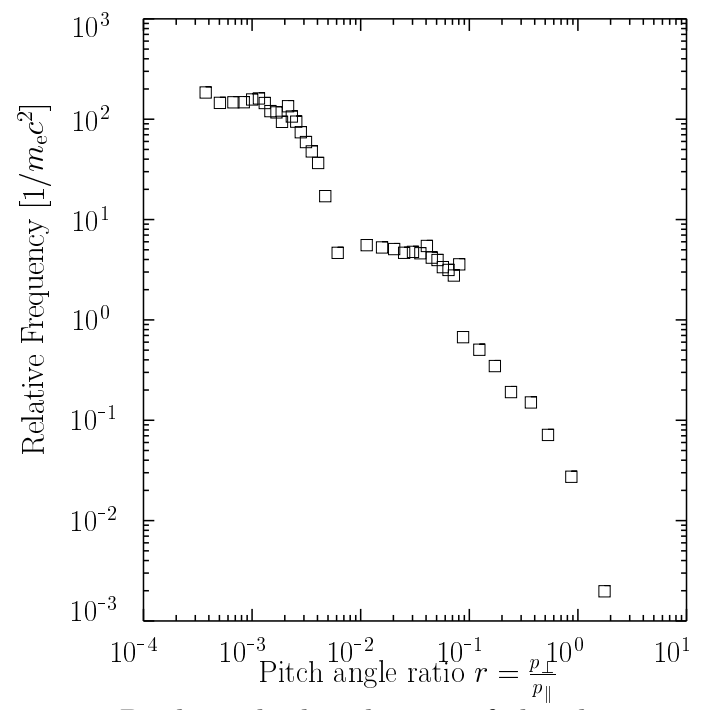

Fig. 11. Pitch angle distribution of the electrons simulated in the realistic 3D-MHD fields.

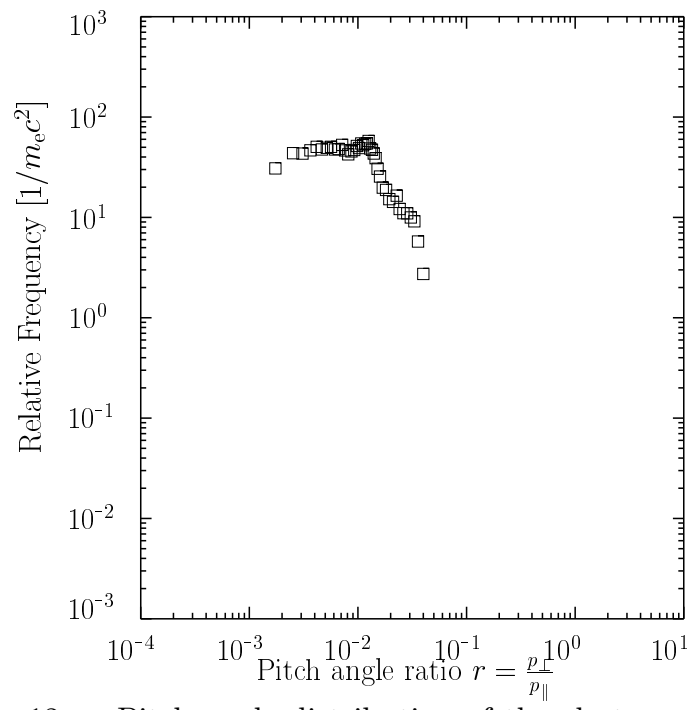

Fig. 12. Pitch angle distribution of the electrons simulated in the oversimplified electric field.

The gyro radius of an accelerated electron is given by

$$
r_{\mathrm{ce}}=v_{\mathrm{Te}} \omega_{\mathrm{ce}}=\frac{v_{\mathrm{Te}} \gamma m_{\mathrm{e}} c}{e B},
$$

which depends on the energy of the electrons $\left(v_{\mathrm{Te}}\right.$ is the thermal velocity of the electrons). Particles are accelerated, in the best case, as long as their gyro radii are smaller than the extent of the resistive region. This criterion gives the maximum energy gain achievable. The effect of considering the loss force (Equ. 13) is shown in Fig. 9. In order to illustrate those effects we overestimate the strength of the loss force by assuming an extremely

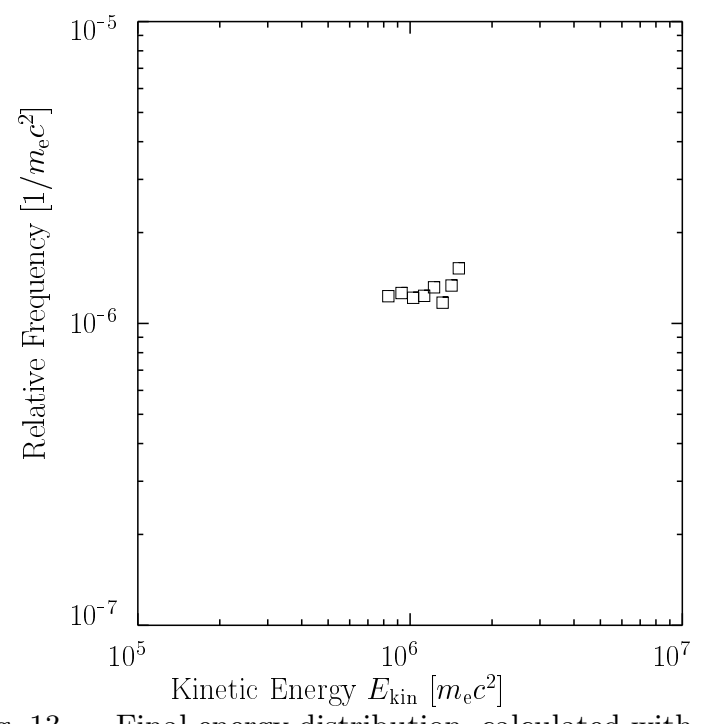

Fig. 13. Final energy distribution, calculated with both, an homogeneous electric field and a homogeneous magnetic field. 
intense photon bath with an energy density $U_{\text {Rad }}$ of about ten times the maximum magnetic energy density $U_{\text {Mag. }}$. Very few electrons are now able to achieve more than $10^{4} m_{\mathrm{e}} c^{2}$. This is the energy, where the loss of energy due to radiative processes balances the energy gain from the electric field. The characteristic features mentioned before remain unchanged apart from the new upper limit for the final energy, which leads to a "pileup" at about $10^{4} m_{\mathrm{e}} c^{2}$.

After all, it can be stated that the usage of rather realistic 3D-MHD fields, i.e. a field configuration that represent evolved reconnection electromagnetic fields rather than analytical idealized fields, gives a rich spectrum displaying a great variety of features. This changes drastically in the case of an oversimplified homogeneous electric field. The next example is calculated with the electric field

$$
\boldsymbol{E}(\boldsymbol{r})=\left(\begin{array}{c}
0 \\
0 \\
E_{z, \max }
\end{array}\right)
$$

where $E_{z \text {, max }}$ denotes the maximum of the $z$ component of the numerical electric field used before. The resulting energy distribution, calculated in the same way as before, is shown in Fig. 10. We should point out that for this simulation nothing has been changed in the magnetic field configuration. Fig. 10 shows a very narrow spectrum around the energy $10^{6} m_{\mathrm{e}} c^{2}$ completely without any feature so characteristic for the spectrum of Fig. 8. First of all, we can clearly see that every electron is accelerated. This unphysical result comes at no surprise, because a constant electric field $E_{z}$ in combination with the strong guiding component $B_{z}$ implies an infinite resistive region. The obvious absence of any structure shows, that the origin of the depression has to lay mainly in the electric field. An oversimplification of $\boldsymbol{E}$ neglects this interesting effect. The enlargement of the resistive region to the whole numerical box also erases the upper limit of acceleration. The maximum energy is given by the potential difference between on side of the box and the other, but that is a pure trivial geometric limit.

In addition, there is no similarity between the rather realistic spectrum Fig. 8 and that calculated with a homogeneous electric field Fig. 10.

Comparison of the pitch angle distribution (Fig. 11) of the realistic simulation with the one of the oversimplified electric field simulation (Fig. 12) also shows a striking difference. While both distributions clearly show that a vast majority of electrons is moving mainly along the magnetic field lines, again a broad spectrum over tree decades and a small amount of electrons, which have a pitch angle greater than $\frac{\pi}{2}$ evolves (Fig. 11). The pitch angle distribution in Fig. 12 on the other side is lacking any hint of the electron population with an pitch angle greater than $\approx 0.01$. An homogeneous electric field can, in fact, not be regarded as a valid approximation of a realistic field configuration.

At this point the question arises, how important is the structure of the magnetic field for the final spectra anyway? In order to answer this question we finally consider the spectrum for

$$
\boldsymbol{E}(\boldsymbol{r})=\left(\begin{array}{c}
0 \\
0 \\
E_{z, \max }
\end{array}\right) \quad \boldsymbol{B}(\boldsymbol{r})=\left(\begin{array}{c}
0 \\
0 \\
B_{z, \max }
\end{array}\right)
$$

This spectrum (Fig. 13) shows a striking similarity with the previously shown one Fig. 10. Both spectra are dominated by the geometry of the numerical box. All of the features seen in Fig. 8 are coming from the complicated structure of the electric field. It seems that the structure of the magnetic field serves only as "stage" for the electrons without significant influence on the spectra. Nevertheless, we should keep in mind, that the $\boldsymbol{B}$ field determines the structure of the $\boldsymbol{E}$ field.

\section{Discussion}

Astrophysical plasmas like the interstellar or intergalactic medium and compact systems like accreting stellar objects are magnetized and highly conducting collisionless media under the influence of external forces. The main driving forces for plasma-magnetic field motions are gravity, rotation and pressure gradients. Due to the lack of significant particle collisions astrophysical plasmas are easily forced to steep magnetic field gradients, which ultimately will evolve into relaxed field configurations via magnetic reconnection. In that sense magnetic reconnection is an unavoidable result of external distortions in collisionless magnetized plasmas. The important observable consequences of reconnection for astrophysical applications follows from the fact that it accounts for heating and particle acceleration by locally converting the stored magnetic energy into bulk heating of the plasma via Ohmic heating or acceleration of a population of high energy particles via magnetic field-aligned electric fields that are associated with the finite conductivity.

The heating capabilities of magnetic reconnection have been investigated in detail, since the heating rate $\propto \eta j^{2}$ is a macroscopic quantity which depends only on the density and temperature, but not on the details of the single particle response to the electromagnetic configuration inside a reconnection zone. In that sense it behaves like a macroscopic transport property. 
The acceleration of particles instead depends on the local properties of the reconnection region. The energization of a charged particle above all depends on the effective electric force the particle "feels". Depending on its initial position and initial energy the particle energy gain differs. Since the only source of information of high energy astrophysics is the electromagnetic radiation, the energy distribution of relativistic particles is one of the most required issues concerning the interpretation of nonthermal radiation sources. Thus, for any acceleration process discussed it is most important to investigate the resulting energy distribution functions in dependence on the various parameters on which the acceleration process depends on. In this context, the twodimensional models published so far for the acceleration of charged particles by magnetic reconnection are not satisfying, since they describe acceleration in linear conductors. They do not describe the effect of the finite extension of the acceleration zone and show always a purely monoenergetic distribution at the maximum energy the particles are "allowed" to reach. The maximum energy in these models is simply given by the product of the homogeneous electric field times the chosen length of the reconnection zone, which is supposed to be a free parameter. This is definitely incorrect; the system size must be finite and must depend on the process responsible for the violation of ideal conductivity.

We have shown the pitfalls of the two-dimensional ansatz and present 3D-simulations of MHD-reconnection plus test particle simulations within the MHD-frame, including radiation losses (synchrotron and inverse Compton scattering). The precise structure of the electric field is the determining factor of the acceleration process in the MHD reconnection region (cf. Sec. V). One has to be extremely careful in simplifying the electric field; in our example the spectrum calculated with a homogeneous electric field is no good approximation to the one that results from a physically motivated field.

Since the investigated magnetic field configuration is a valid $3 \mathrm{D}$ generalization of a $2 \mathrm{D} \mathrm{X}$-type field the usage of an homogeneous electric fields in X-type electric fields should also be misleading as far as the spectrum is concerned.

Whereas particle acceleration in a simplified X-type configuration with a homogeneous electric field is an interesting subject of it's own, it is obviously not appropriate for the study of particle acceleration in magnetic reconnection zones. We have shown that for this task we need much more realistic fields and at least numerical approaches in future should use 3D-MHD fields. A further extension of the work presented in this contribution will be the consideration of multiple $\mathrm{X}_{-}$line and O-point reconnection configurations (c.f 19 and 13 for the $2 \mathrm{D}$ case) which should show a larger class of different kinds of particles populations.

The strong influence of radiative losses on the resulting energy distribution of the accelerated charged particles, even without overestimation of the photon density, gives us the clear indication that we should not restrict ourselves to the use of realistic field configurations alone. By not taking into account SY and/or IC losses in relativistic particle acceleration processes in reconnection zones the particle pile-up at the upper energy limit is completely neglected and the maximum energy of the particles is incorrectly estimated. This shows that the consideration of loss processes should be obligatory for the determination of quantitative results in the relativistic case. In this context we briefly note that particle acceleration by 3D-reconnection has considerable observational consequences. As is obvious from our simulations 3D-reconnection produces power-law energy distributions, which would emit power-law radiation spectra, i.e. decreasing radiation flux with increasing frequency $\nu$ : Flux $\propto \nu^{-\alpha}$. For synchrotron and inverse Compton emission the index of the energy distribution $p$ is related with the spectral index $\alpha$ via $p=2 \alpha+1$ In contrast the simple analytical models with a constant electric field give mononergetic energy distributions which give synchrotron spectra where the received radiation flux increases with increasing frequency up to some critical frequency determined by the energy of the accelerated particles (Flux $\propto \nu^{1 / 3}$ for an optically thin monoenergetic source) (c.f 44 ).

At the present time we do not consider any interaction between particles and plasma waves and we neglect losses due to pair production. These effect should be addressed in future simulation studies as well as the interplay of second order magnetic fields induced by accelerated particle beams and the ponderomotive force of the excited plasma fluctuations. We feel that such studies will prove to be very helpful to clarify the role of reconnection in a variety of dynamical astrophysical plasma phenomena as different as, e.g., extragalactic jets, TeV burst and solar flares.

\section{Acknowledgments}

This work was supported by the Deutscher Akademischer Austausch Dienst (DAAD) through the grant D/98/02999, the Alexander von Humboldt foundation through the Feodor Lynen program (GTB) and the Deutsche Forschungsgemeinschaft through the Schwerpunkt "Physik der Sternentstehung" and the grant Le 1939-4/1. 
1. J.G. Kirk, Plasma Astrophysics (Springer, Berlin, 1994) p. 225 .

2. J. Kuijpers, Lect. Notes Phys. 469, 101 (1996).

3. V.L. Ginzburg and S.I. Syrovatskii, The Origin of Cosmic Rays (Pergamon Press, Oxford, 1964).

4. R.D. Blandford and J.P. Ostriker, Astrophys. J. 221, L29 (1978).

5. E. Fermi, Phys. Rev. Lett. 75, 1169 (1949).

6. R. Ramaty and R.J. Murphy, Space Sci. Rev. 45, 213 (1987).

7. R. Schlickeiser, Astrophys. J. 336, 264 (1989).

8. H. Alfvén, Cosmic Plasma, (Reidel, Dordrecht, 1981).

9. K. Schindler, M. Hesse, and J. Birn, Astrophys. J. 380, 293 (1991).

10. E.R. Priest, Solar Magnetohydrodynamics, (Reidel, Dordrecht, 1987).

11. A. Otto and G.T. Birk, Geophys. Res. Lett. 20, 2833 (1993).

12. H. Lesch and G.T. Birk, Astron. Astrophys. 324, 461 (1997).

13. A. Kopp, G.T. Birk, and A. Otto, Planet. Space Sci. 46, 405 (1998).

14. T.W. Speiser, J. Geophys. Res. 70, 4219 (1965).

15. S.W.H. Cowley, Planet. Space Sci. 27, 539 (1978).

16. H.-J. Deeg, J.E. Borovsky, and N. Duric, Phys. Plasmas B 3, 2660 (1991).

17. R.W. Moses, J.M. Finn, and K.M. Ling, J. Geophys. Res. 98, 4013 (1993).

18. G.E. Vekstein and E.R. Priest, Phys. Plasmas 2, 3169 (1995)

19. B. Kliem, J. Schumacher, and D.R. Shklyar, Adv. Space. Res. 21, 563 (1998).

20. Y.E. Litvinenko and B.V. Somov, Sol. Phys. 146, 127 (1993).

21. R.L. Kaufmann, C. Lu, and D.J. Larson, J. Geophys. Res. 99, 11277 (1994).

22. Y. Litvinenko, Astrophys. J. 462, 997 (1996).

23. R.D.Bentley and J.T. Mariska, Magnetic Reconnection in the Solar Atmosphere, Conference Series Volume 111 (Astronomical Society of the Pacific, San Francisco1, 1997)

24. Akasofu, S.-I. and J.R. Kan, Physics of Auroral Arc Formation, (American Geophysical Union, Washington, 1981).

25. J. Birn, M.F. Thomsen, J.E. Borovsky, G.D. Reeves, D.J. McComas and R.D. Belian, J. Geophys. Res. 102, 2325 (1997).

26. J. Birn, M.F. Thomsen, J.E. Borovsky, G.D. Reeves, D.J. McComas and R.D. Belian, J. Geophys. Res. 103, 9235 (1998).

27. S.V. Bulanov and P.V. Sasorov, Sov. Astron. Let., 19, pp. 464-468, (1975).

28. S.V. Bulanov, Sov. Astron. Let. 6, pp. 206-208, (1980).

29. M.V. Nocera, F. Pegoraro, S.V. Bulanov, and G. Bertin, Physica Scripta, T63, pp. 197-202, (1996).
30. P. Démoulin, J.C., Hénous, E.R. Priest and C.H. Mandrini, Astron. Astrophys. 308, 643 (1996).

31. G. Hornig and L. Rastätter, Phys. Scripta T74, 34 (1998).

32. G.T. Birk and A. Otto, J. Atm. Sol.-Terr. Phys. 59, 835 (1997).

33. A. Otto, Comp. Phys. Com. 59, 185 (1990).

34. G.T. Birk and A. Otto, Adv. Space Res.20, 1301 (1997).

35. J.D. Huba, Unstable Current Systems and Plasma Instabilities in Astrophysics (Dordrecht, Reidel, 1985), p. 315 .

36. V.M. Vasyliunas, Rev. Geophy. Space. Phys. 13, 303 (1975).

37. K. Schindler, M. Hese, and J. Birn, J. Geophys. Res., 93, 5547 (1988).

38. F.S. Mozer, Physics of Auroral Arc Formation (American Geophysical Union, Washington, D.C., 1981), pp. 136-142.

39. W.H. Press, B.P. Flannery, S.A. Teukolsky, and W.T. Vetterling, NUMERICAL RECIPES in C (Cambridge University Press, Cambridge UK, 1988).

40. R. Schopper, H. Lesch, and G.T. Birk, Astron. Astrophys., 335, pp. 26-32 (1998).

41. L.D. Landau, E.M. Lifshitz, The Classical Theory of Fields (Addison-Wesley Publ. Comp., Reading Mass. USA, 1951).

42. R.D. Blandford, In Active Galactic Nuclei, Saas-Fee Advanced Course 20, (Springer, Heidelberg, 1990), p. 161.

43. J. Schumacher and B. Kliem, Phys. Plasmas 3, 4703 (1996).

44. M.S. Longair, High energy astrophysics (Cambridge University Press, Cambridge UK, 1981). 